\title{
New metastable carbon phases observed by HRTEM
}

\author{
Duan Luo ${ }^{1,2,3}$, Liuxiang Yang ${ }^{4,5}$, Hongxian $\mathrm{Xie}^{6}$, Ho-kwang Mao ${ }^{4,5}$, and Jianguo Wen ${ }^{1}$ \\ 1. Center for Nanoscale Materials, Argonne National Laboratory, Argonne, IL 60439 \\ 2. Key Laboratory of Ultra-fast Photoelectric Diagnostics Technology, Xi'an Institute of Optics \\ and Precision Mechanics, Chinese Academy of Sciences, Xi'an 710119, China. \\ 3. University of Chinese Academy of Sciences, Beijing 100049, China. \\ 4. Center for High Pressure Science and Technology Advanced Research, Beijing, 100094, China \\ 5. Geophysical Laboratory, Carnegie Institution of Washington, Washington, DC, 20015, USA \\ 6. Hebei University of Technology, Tianjin 300132, China \\ *dluo@anl.gov
}

The mutual phase transformation between various carbon allotropes such as graphite, diamond and carbon onions has been of tremendous scientific and technological interest for more than 70 years since these polymorphs have different electronic properties and mechanical performances. The search for novel metastable crystalline and amorphous phases of carbon, especially related to the transition of graphite to diamond, is crucial for understanding their transformation mechanisms. Furthermore, synthesis of such metastable phases may have a significant impact on practical applications due to their outstanding properties, for example, the metastable hexagonal diamond (HD) is predicted to be about $60 \%$ harder than cubic diamond (CD) [1].

In this study, by using aberration-corrected high resolution TEM (HRTEM) technique, we experimentally observed several new metastable phases different from graphite and diamond on the atomic scale. At ambient conditions, hexagonal graphite $(\mathrm{HG})$ is the most stable form of carbon, whereas CD is a metastable phase. However, the direct graphite-to-diamond transition is limited by a large activation barrier. Rhombohedral graphite $(R G)$ was widely considered as an intermediate phase of such conversion $[2,3]$, but rare experimental evidence was reported. Using single crystal graphite as starting material, we found RG in the high temperature and high pressure (HTHP) samples (Fig. 1). Instead of AB stacking of HG, it is ABC stacking with the similar interplanar separation of HG. Furemore, we discovered large amount of mixture of hexagonal and cubic diamond with different stacking sequences (Fig. 2). Those new forms of diamond structure are expected to provide new insights into the mutual transformation among HG, HD and CD [4]. The phase transformation mechanism among these metastable phases will be studied using ultrafast electron diffraction [5].

References:

[1] Z. Pan, H. Sun, Y. Zhang, and C. Chen, Physical review letters, 102(5), (2009), p. 055503.

[2] S. Scandolo, M. Bernasconi, G. L. Chiarotti, P. Focher, E. Tosatti, Physical Review Letters, 74, (1995), p. 4015.

[3] R. Z. Khaliullin, H. Eshet, T. D. Kühne, J. Behler, M. Parrinello, Nature materials, 10, (2011), p. 693.

[4] H. He, T. Sekine, T. Kobayashi, Applied physics letters 81 (2002), p. 610.

[5] Electron microscopy was performed at the Center for Nanoscale Materials at Argonne National Laboratory, a U.S. Department of Energy Office of Science Laboratory operated 
under Contract No. DE-AC02-06CH11357 by U. Chicago Argonne, LLC. D.L. acknowledges the financial support of the China Scholarship Council (CSC) for studying abroad.
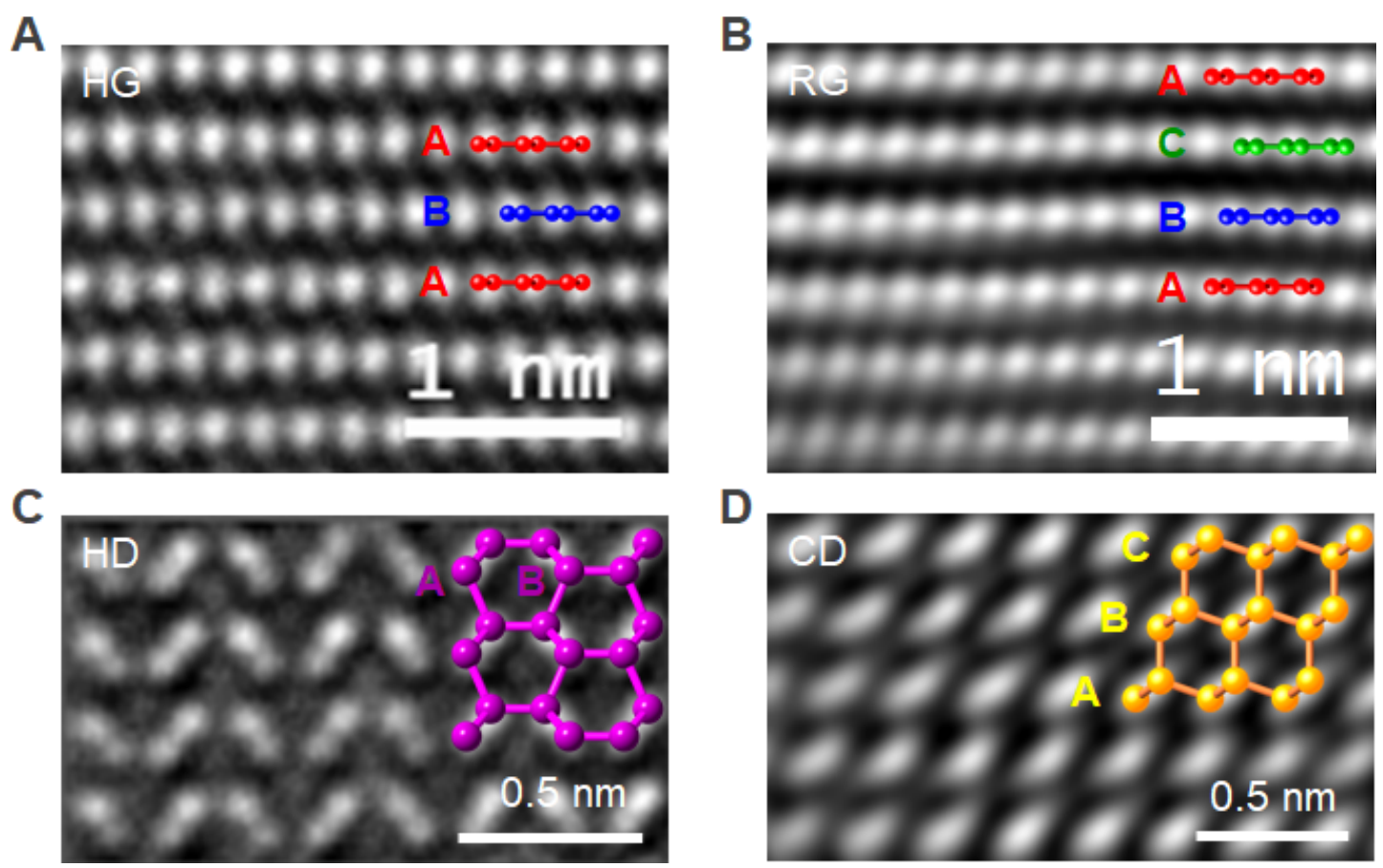

Figure 1. High-resolution TEM images of (a) hexagonal graphite (b) rhombohedral graphite (c) hexagonal diamond, and (d) cubic diamond phases.
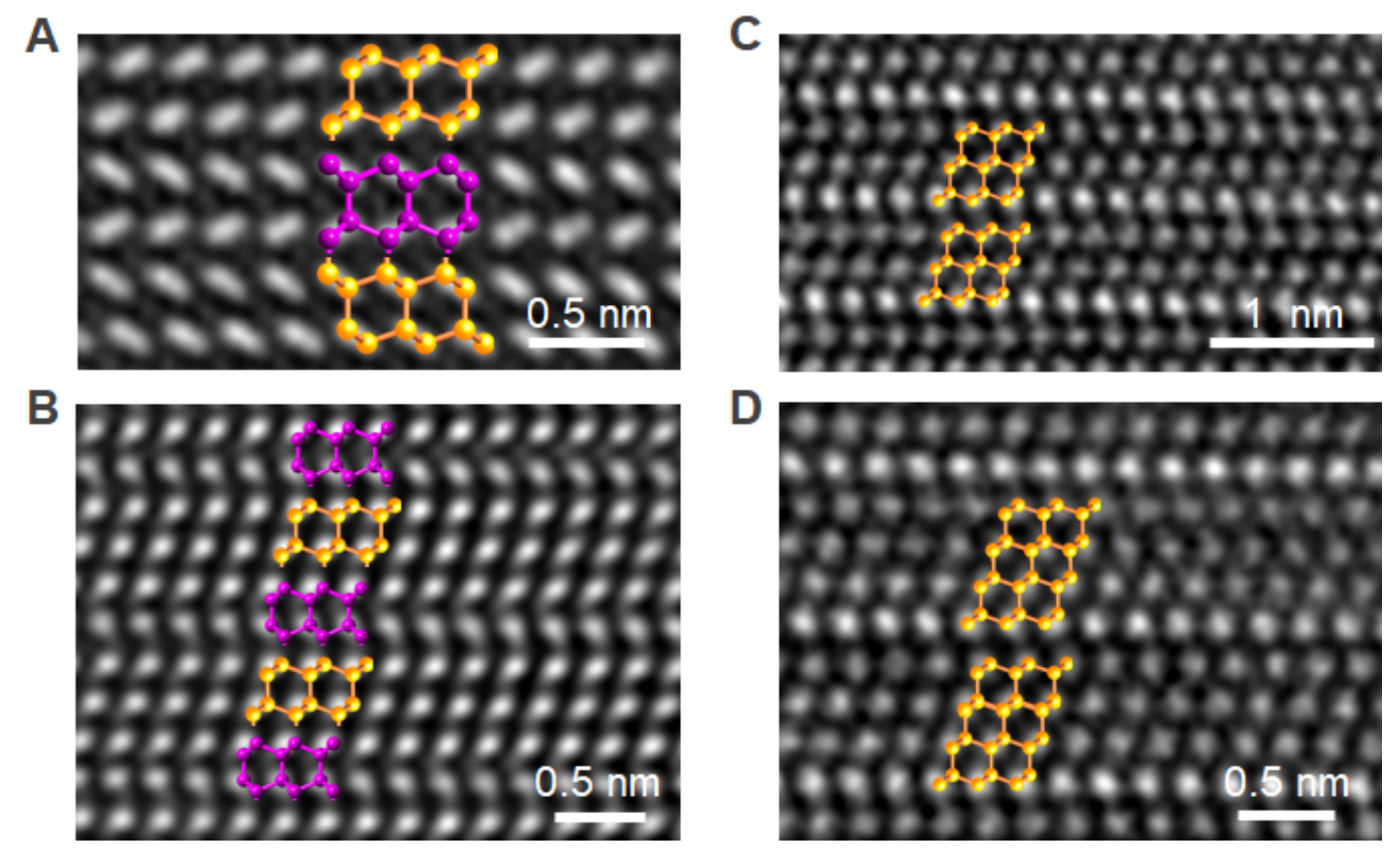

Figure 2. HREM images showing different stacking sequences of diamond. 